\title{
Muscularis Propria
}

National Cancer Institute

\section{Source}

National Cancer Institute. Muscularis Propria. NCI Thesaurus. Code C62404.

The detrusor muscle of the bladder, forming the muscular wall of the bladder, consisting of the inner longitudinal; middle circular; and outer longitudinal layers. 